\title{
The application of Simulation (Virtual Reality) for Safety Training in the Context of Mining Industry
}

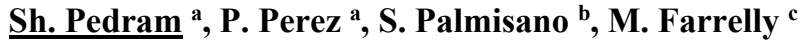 \\ a SMART Infrastructure Facility, School of Engineering, University of Wollongong, NSW \\ ${ }^{b}$ School of Psychology, University of Wollongong, NSW \\ ${ }^{c}$ Mines Rescue, Coal Services, Woonona NSW \\ Email: spedram@uow.edu.au
}

\begin{abstract}
Virtual Reality (VR) technology has been used to train for various operations and dangerous circumstances where it is believed that training objectives cannot be achieved easily or the cost will prohibitive. Van Wyk and colleagues (2009) define VR-based training environments as "real-time computer simulations of the real world, in which visual realism, object behavior and user interaction are essential elements". The use of VR-based training environments assumes that Human-Machine interaction stimulates learning processes through better experiencing and improved memorization, leading to a more effective transfer of the learning outcomes into workplace environments. However, there are many human factors (internally and externally), which have impact on the quality of the training and learning process which need to be identified and investigated.
\end{abstract}

In this article, initially factors affecting the quality of the training and learning process for underground mine rescuers have been identified and then measured by using pre- and post-training questionnaires. Then statistical analyses have been performed to investigate the relationship among trainees' perceived realism, usefulness and success. Also, trainers' perception on 360-VR usefulness and success has been measured and compared with trainees. As the result of analysis indicated, trainees typically found the training sessions useful and perceived them to be successful; many felt that it was not really consistent with their real life experience. It would appear that perceived usefulness plays important role in forming the perception of success with high correlation and that the level of realism is not necessarily a deciding factor. Also, there was no significant difference between perceived usefulness and success between trainees and trainers. This research was conducted in collaboration with Mines Rescue Pty Ltd (a training provider for the coal mining industry in NSW, Australia) and was focused on training programs developed for mine rescue brigades. Data was collected from 94 mine rescue brigades (trainees) who attended a 360-VR training session over a twelvemonth period and 25 trainers who run the training sessions.

Keywords: Virtual Reality (VR), safety training, evaluation, mining industry 
Pedram et al., The application of Simulation (Virtual Reality) for Safety Training in the Context of Mining Industry

\section{INTRODUCTION}

As 'virtual reality' became a widely adopted terminology to describe immersive simulations, early studies demonstrated that skill acquisition through VR-based training was dependent upon the task to be trained, as well as the amount and type of training (Hays et al., 1992). Other studies compared the quality of VR-based training outcomes with traditional programs (classroom and flying lessons). High risk industries progressively adopted VR environments as a means to address some of the limitations associated with traditional onsite and classroom training (Bliss et al., 1997, Tichon and Burgess-Limerick, 2011). Although onsite training for high risk industries maximises the fidelity of the experience, it also conveys two issues: cost and safety. Besides, onsite training often competes against scheduled activities of the plant or the mine, limiting training opportunities. Therefore, VR-based training is seen as a promising and complementary option to traditional approaches whereby trainees can be exposed to various and often extreme scenarios in a safe, replicable and cost-effective environment. Burdea and colleagues (2003) categorize the main characteristics of VR technologies as '3Is': Immersion, Interaction and Imagination.

Moreno and Mayer (2007) propose three elements of interaction to characterise VR technologies, i) the physical element (the feeling that you are actually in the replication of physical world), ii) the social element (the feeling that you are sharing the experience with someone else) and iii) the self-presence element (seeing virtual version of yourself). The main purpose behind interaction is to construct the knowledge and adding information to the learner's memory. Unlike more passive computer-aided training approaches (videos or webinars), VR-based training provides an intimate level of interaction between trainees and the learning content, allowing for more flexible problem-solving and decision-making processes. Shifting from batch simulation to VR technology may cost more investment but allows to evaluate more possibilities and options. Additionally, providing feedback is also a form of interaction. Moreno and colleagues (2002) state that there are two forms of feedback: "corrective feedback", where the trainee will be informed whether he/she was right or wrong, and "explanatory feedback", where the explanation is given why he/she was right or wrong. Their study shows that trainees who received explanatory feedback performed better in solving complex problems compared to the other group who just received the corrective feedback. Moreover, Fox and colleagues (2009) in their studies focused on immersion and define immersion as: "The psychological experience of losing oneself in the digital environment and shutting out cues from the physical world is known as immersion". VR learning environments have been categorized based on their level of immersion by Moreno and Mayer (2002): (i) no immersion (such as illustrated text), (ii) medium immersion (such as games and computer displays) and (iii) high immersion (such as head-mounted displays). High degrees of immersion increase the sense of presence which might lead to more engagement and deeper learning compared to approaches where trainees remain observers (Salzman et al., 1999). Interest theory (Salzman et al., 1999) states that the higher the sense of presence trainees feel will cause higher motivation which might motivate trainees to work harder to grasp deeper learning. On the other hand, interference theory states that highly immersive environments might overload trainees with too much of information (Moreno and Mayer, 2002).

\section{VR AS A TRAINING TOOL}

VanWyk and colleagues (2009) define VR-based training environments as "real-time computer simulations of the real world, in which visual realism, object behavior and user interaction are essential elements".

Educational psychologists often argue that human learning involves the construction of new knowledge based on prior information. For instance, according to Dewey and colleagues (1985), active experiences (such as interactions between learners and the environment) could lead to the construction of new knowledge. In this regard, educators and trainers can be seen as facilitators (Hunkins and Ornstein, 1998) who help trainees shape their learning experience and promote learning. Achieving an acceptable level of competency is the main purpose of training programs. Learning occurs as a result of practice (i.e. the act of repeating an action). Causal queues and repetitions are meant to stimulate individual experiences and to enrich corresponding mental models (Jou and Wang, 2012). Ultimately, this iterative learning in context should limit the number of human errors to a tolerable level as skill sets dramatically improve(Deaton et al., 2005).VR technology has been used to train for various operations and dangerous circumstances where it is believed that training objectives cannot be achieved easily or the cost will prohibitive. The use of VR-based training environments assumes that Human-Machine interaction stimulates learning processes through better experiencing and improved memorization, leading to a more effective transfer of the learning outcomes into workplace environments. As stated by Meadows (2001): "When I hear, I forget; when I see, I remember; when I do, I understand". Fulton and colleagues (2011) argue that interactive models like flight simulators are designed to improve the trainee's understanding of the consequences of decisional queues under limited 
Pedram et al., The application of Simulation (Virtual Reality) for Safety Training in the Context of Mining Industry

resource availability (material, time or energy) and uncertain or hazardous conditions (unintended consequences). Seymour and colleagues argue that the more realistic the experience is, the stronger the learning process. In situations where real life training opportunities are limited, hazardous or impossible, like emergency responses, virtual reality simulators offer the opportunity to emulate many wide-ranging experiments (Seymour et al., 2002). For example, mining industries around the world have also begun implementing VR technology to train their workforce for various operations such as mining equipment operation including dozers, dragline, longwall or conveyer belt (Wilkes, 2001). However, there is no systematic evaluation result to indicate to which extend these VR-based training programs were successful in delivering long-lasting competency (Tichon and Burgess-Limerick, 2011). A VR environment, called MinerSIM, was developed by Nutakor (2008) at university of Missouri in US to train miners to install rock bolts. The training package includes an online tutorial and a virtual reality simulator. To date, the usability of web tutorial is the only aspect that has been evaluated. In another study, Lucas and Thabet (2008) evaluated the use of a virtual conveyer belt to train miners for mining equipment safety. One group of miners was trained using a desktop and the other group used immersive VR technology. There wasn't any significant difference between the groups, partly due to the very small sample in use (12 trainees in total).

This paper focuses on evaluation of VR training environment for safety training in mining industry. Patton (1997) defined evaluation as: "the systematic collection of information about the activities, characteristics, and outcomes of programs to make judgements about the program, improve program effectiveness, and/or inform decisions about future programming". As a consequence, we propose to develop an in-situ evaluation framework that will assess groups of trainees in their usual training environment, using non-disruptive subjective information. The idea is to let the trainer conduct a VR-based training session as usual without distracting the trainees and to embed our evaluation into an annual cycle of training.

\section{PARTICIPANTS AND STUDY CONTEXT}

\subsection{Technology-in-use}

The research was conducted in collaboration with Mines Rescue Pty Ltd, a training provider for the coal mining industry in Australia that operates four training stations in New South Wales (Woonona, Lithgow, Newcastle, Singleton and Woonona). Each centre delivers classroom, onsite and VR-based training programs. Our study focussed on training programs developed for the mine rescue brigades. These brigades are made of five to seven highly specialized volunteers who act as primary responders in case of major mining incidents or accidents. The methodological framework was designed and tested at Woonona station. This paper focuses exclusively on the training programs developed for the 360 degree immersive theatre (360-VR). The $360-\mathrm{VR}$ is a $10 \mathrm{~m}$ diameter, $4 \mathrm{~m}$ high cylindrical screen that displays a $3 \mathrm{D}$ stereo, 360 degree virtual environment, providing a fully immersive experience to participants equipped with $3 \mathrm{D}$ glasses.

\subsection{Training Scenario}

Before rescue brigades entering the 360-VR, they wear their BG4 suits and they go under oxygen. Then, rescue brigades were debriefed by trainer on the accident: "The mine has been operating under its Level 1 trigger. This allows mining to continue on a normal basis. At the end of the last shift at $6.57 \mathrm{am}$ an alarm was activated on the surface monitoring system at the monitoring point $\mathrm{M}$. A CO reading of between 30 and 40 PPM has been recorded since that time from the real time monitoring point. Results show no traces of hydrogen or ethylene from the sampling point and this has been confirmed. The control officer was unable to contact the longwall deputy who had already departed for the surface. The crew has now been held back awaiting an inspection to verify the source of the elevated readings." The large area within the theatre allows for a mixed reality experience, with small groups of trainees (5 to 7) able to interact both with props (virtual gas detectors) and with each other, in order to ensure that appropriate responses, activities and reflexes are included as part of the training experience. The trainer (Fig. 1) guides the trainees through successive stages of the scenario, prompting them for appropriate actions or responses. 
Pedram et al., The application of Simulation (Virtual Reality) for Safety Training in the Context of Mining Industry
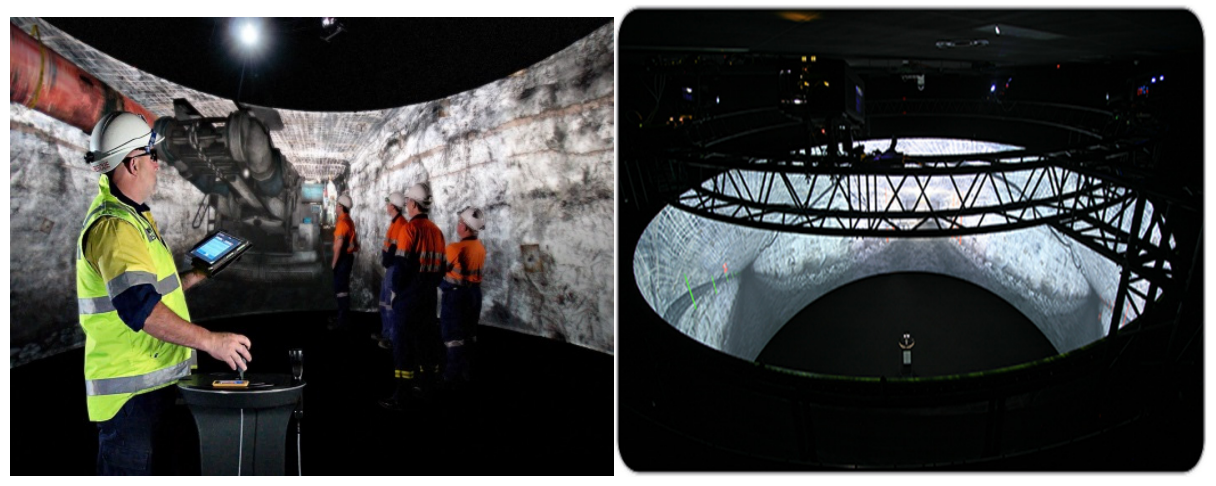

Figure 1. 360-VR training (Coal services Pty. Ltd)

\subsection{Participants}

Between March and July 2015, 94 trainees interviewed for this study and all of the participants in the study were male, aged between 24 and 64 years, with their time spent in mining and mines rescue ranging from between 5 and 40 years. The participants in this study were 94 experienced underground miners who had volunteered to join the rescue brigade. Also, 25 trainers have been interviewed who were in running the training courses in $360-\mathrm{VR}$.

\section{METHODOLOGY}

Since the objective of this study is to evaluate the role of $360-\mathrm{VR}$ for safety training it was not possible to measure performance and training outcome. Therefore we chose to use users' opinion technique where users are asked to give their opinions on the conducted training, even though this technique does not reflect on knowledge creation and training transfer (Nutakor, 2008) but it allows us to understand the factors affecting the training process in 360-VR. The researcher attended all the 360-VR training sessions to observe trainees experiences. She also distributed the questionnaires directly before and after these training sessions. The pretraining questionnaire was distributed to participants prior to attending the 360-VR training. The aim of this questionnaire is to measure the trainees' state of mind and experience with technology prior the training. After the VR training, the post-training questionnaire was distributed to measure the participants' learning and experiences as a user. Other key questions asked about the participants' perception of the perceived level of realism, the success and the usefulness of the VR training. Primary data was obtained using Likert Scale based Questionnaires. Our pre- and post-training questionnaires were based on items taken from established questionnaires.

Pre-training questionnaire included items from: Game Experience Measure (GEM): trainee's prior experience with computers and video games based on (Taylor and Barnett, 2011), Immersive Tendencies (ITQ): sense of focus, involvement and alert prior training session based on (Witmer and Singer, 1998), Simulator Sickness (SSQ): history of motion or simulator sickness (nausea, disorientation and oculomotor symptoms) and self-assessment prior training based on (Kennedy et al., 1993), Dundee Stress State (DSSQ): sense of engagement, distress or worry prior training session based on (Matthews et al., 1999) and Intrinsic Motivation Inventory (IMI): sense of motivation, confidence and competition prior training session based on(McAuley et al., 1989).

And post-training questionnaire included items from: Simulator Sickness (SSQ): sense of sickness during session (Kennedy et al., 1993), User Interface (UIQ): easiness to use and perceived realism based on (Taylor and Barnett, 2011), Game Engagement (GEQ): sense of engagement with scenario and environment based on (Taylor and Barnett, 2011), Involvement and Presence (IPQ): sense of presence and involvement during session based on (Witmer and Singer, 1998), Intrinsic Motivation Inventory (IMI): enjoyment and motivation during session based on (Witmer and Singer, 1998), Immersive Tendencies (ITQ): sense of focus and immersion during session based on (Witmer and Singer, 1998), Dundee Stress State (DSSQ): sense of pressure or tension during session based on (Matthews et al., 1999). Due to limits on our testing time (which prevented use of the full questionnaires) key items taken from standard questionnaires to measure each factor of interest. These factors had been identified by previous studies as being important for the success of VR training. In order to check that each group of items was still measuring the same factor (i.e. as the original full questionnaires), ensured that the Cronbach's Alpha value for each factor was above 0.7. 
Pedram et al., The application of Simulation (Virtual Reality) for Safety Training in the Context of Mining Industry

\section{RESULTS}

Table (1) and (2) summarise the mean values for each of the pre-training and post-training factors where Likert scales ranging from highly disagree (0) to highly agree (5). Even though trainees had limited gaming experience $(M=1.4)$ but they have been motivated $(M=4.2)$ to attend the training. Moreover, they reported better than average scores for "ease of use" $(M=3.6)$, "enjoyment" $(M=3.8)$, "presence" $(M=3.3)$, "usefulness" (M=4.09) and perceived learning $(\mathrm{M}=3.5)$ (scores out of 5).

Table 1. Mean value for Pre-training factors

\begin{tabular}{|c|c|c|c|c|}
\hline Type & Factor & Lower & Mean & Upper \\
\hline Pre Training & Gaming Experience & 1.3137 & 1.4185 & 1.5179 \\
\hline Pre Training & $\begin{array}{l}\text { Sense of Alert and } \\
\text { Presence }\end{array}$ & 3.8348 & 3.9427 & 4.0505 \\
\hline Pre Training & Sense of Stress & 2.5461 & 2.7312 & 2.9162 \\
\hline Pre Training & Sense of Motivation & 4.1142 & 4.2007 & 4.2873 \\
\hline Pre Training & $\begin{array}{l}\text { Sense of Confidence and } \\
\text { Competency }\end{array}$ & 3.8454 & 3.9355 & 4.0256 \\
\hline Pre Training & Sense of Worry & 2.6344 & 2.828 & 3.0215 \\
\hline Pre Training & Sense of Competition & 3.021 & 3.2151 & 3.4091 \\
\hline Pre Training & Sickness & 1.3285 & 1.359 & 1.3895 \\
\hline
\end{tabular}

Table 2. Mean value for Post-training factors

\begin{tabular}{|l|l|r|r|r|}
\hline Type & Factor & \multicolumn{1}{l|}{ lower } & \multicolumn{1}{l|}{ Uean } & Upper \\
\hline Post Training & $\begin{array}{l}\text { Sense of Engagement } \\
\text { and Interaction }\end{array}$ & 3.3292 & 3.4663 & 3.6034 \\
\hline Post Training & Sense of Ease of Use & 3.5325 & 3.6957 & 3.8588 \\
\hline Post Training & Sense of Fatigue & 2.1646 & 2.3258 & 2.4871 \\
\hline Post Training & Sense of Enjoyment & 3.803 & 3.8966 & 3.9902 \\
\hline Post Training & Sense of Stress, & 2.1497 & 2.2317 & 2.3138 \\
\hline Post Training & Sensure and tension & 3.1904 & 3.3015 & 3.4126 \\
\hline Post Training & Sense of Realism & 2.8941 & 3.1989 & 3.5036 \\
\hline Post Training & Sense of Usefulness & 3.9517 & 4.092 & 4.2322 \\
\hline Post Training & Success & 3.201 & 3.401 & 4.102 \\
\hline Post Training & Sickness & 1.3237 & 1.3931 & 1.4626 \\
\hline Post Training & Perceived Learning & 3.3467 & 3.5301 & 3.7136 \\
\hline
\end{tabular}

As Table (3) indicates the mean value of perceived level of realism, usefulness and success from trainees' point of view we decided to perform Cross-tabulations to better understand the success of 360-VR as a training tool. Cross-tabulations between "perceived realism" and "perceived success" and between "perceived realism" and "perceived usefulness" showed that while trainees typically found the training sessions useful and perceived them to be successful, many felt that it was not really consistent with their real life experience. It would appear that perceived usefulness plays important role in forming the perception of success with high correlation $(\mathrm{r}=.609, \mathrm{P}<.05)$ and that the level of realism is not necessarily a deciding factor $(\mathrm{r}=.356, \mathrm{P}<.05)$.

Table 3. Mean value of Perceived level of realism, usefulness and Success form trainees point of view (from Left to right)

\begin{tabular}{|l|l|r|}
\hline \multirow{2}{*}{$\mathrm{N}$} & Valid & 86 \\
\cline { 2 - 3 } & Missing & 7 \\
\hline Mean & & 2.7674 \\
\hline \multicolumn{2}{|l|}{ Mode } & 3.00 \\
\hline
\end{tabular}

\begin{tabular}{|l|l|r|}
\hline \multirow{2}{*}{$N$} & Valid & $\mathbf{8 7}$ \\
\cline { 2 - 3 } & Missing & $\mathbf{6}$ \\
\hline Mean & Mode & 4.0920 \\
\hline \multicolumn{2}{|l|}{ Mor } \\
\hline
\end{tabular}

\begin{tabular}{|l|l|r|}
\hline \multirow{2}{*}{$\mathbf{N}$} & Valid & $\mathbf{8 6}$ \\
\cline { 2 - 3 } & Missing & $\mathbf{7}$ \\
\hline Mean & 3.2326 \\
\hline \multicolumn{2}{|l|}{ Mode } & 3.00 \\
\hline
\end{tabular}

Additionally, the result of Cross-tabulations indicated 59 out of 85 trainees found the 360-VR session to be useful and $32 / 85$ stated $75-100 \%$ it was successful as a training environment to deliver content.

Additionally, trainers have been surveyed to identify from their point of view to what extend 360-VR was useful and successful as a training tool (Table 4). 
Pedram et al., The application of Simulation (Virtual Reality) for Safety Training in the Context of Mining Industry

Table 4. Mean value of perceived usefulness and success from trainers' point of view (from Left to Right)

\begin{tabular}{|l|l|r|}
\hline \multirow{2}{*}{$\mathrm{N}$} & Valid & $\mathbf{2 5}$ \\
\cline { 2 - 3 } & Missing & $\mathbf{0}$ \\
\hline \multicolumn{2}{|l|}{ Mean } & 4.3200 \\
\hline \multicolumn{2}{|l|}{ Mode } & 4.00 \\
\hline
\end{tabular}

\begin{tabular}{|l|l|r|}
\hline \multirow{2}{*}{$N$} & Valid & $\mathbf{2 5}$ \\
\cline { 2 - 3 } & Missing & $\mathbf{0}$ \\
\hline \multicolumn{2}{|l|}{ Mean } \\
\hline \multicolumn{2}{|l|}{ Mode } & 3.4000 \\
\hline
\end{tabular}

To understand whether trainees' perception, in regards to success and usefulness of 360-VR as a training toll differs from trainers' point of view, initially we performed hapiro-Wilk test which revealed that the Sig.= .000 meaning the distribution of the data is not normal therefore we performed Mann-Whitney test. By doing Mann-Whitney test analysis we are comparing the distribution of the ranks between two groups of trainees and trainers. Mann-Whitney test relies on scores being ranked from lowest to highest; therefore, the trainees group with the lowest mean rank is the group with the greatest number of lower scores in it. Similarly, the trainers group with the highest mean rank have greater number of high scores within it.

As Table (5) indicates, trainees and trainers did not differ in their perception of usefulness of VR as a training environment with $Z=-1.563 ; p=.118>.05$. Moreover, as the rank table shows (Table 5) average of the Usefulness scores of the trainees was 54.37, while the trainers have score rank average of 63.90. The close rank averages of the groups' perceived usefulness scores indicate that both groups had somewhat equal understanding and perception about VR environment being useful as a training tool.

Table 5. Mann-Whitney Test statistics table (on left side) and Rank Table (on right side) to compare perceived usefulness between trainees and trainers

\begin{tabular}{|l|r|l|l|r|r|r|}
\hline & \multicolumn{1}{|c|}{$\begin{array}{c}\text { Perceived } \\
\text { Usefulness }\end{array}$} \\
\cline { 2 - 6 } & 902.500 \\
\cline { 2 - 7 } Mann-Whitney U & 4730.500 \\
\hline Wilcoxon W & -1.563 \\
\hline$Z$ & .118 & Perceived & Group & N & Mean Rank & \multicolumn{1}{c|}{ Sum of Ranks } \\
\hline Asymp. Sig. (2-tailed) & Trainer & 25 & 63.90 & 1597.50 \\
\cline { 2 - 7 } & & Trainee & 87 & 54.37 & 4730.50 \\
\cline { 2 - 7 } & & Total & 112 & & \\
\hline
\end{tabular}

Additionally, Mann-Whitney test has been conducted to investigate whether trainees and trainers differ in their perception of the success of the $360-\mathrm{VR}$ as a training tool. An examination of the findings in Table Test statistics (Table 6) reveals the results of Mann Whitney U test for the perceived usefulness of the trainees and trainer groups did not show any significant statistical difference $(Z=-0.967 ; p=.333>.05)$. The rank table (Table 6) average of the Success scores of the trainees was 54.56, while the trainers have score rank average of 60.94. The close rank averages of the groups' perceived success scores indicate that both groups had somewhat equal understanding and perception about VR environment being successful as a training tool.

Table 6. Mann-Whitney Test statistics table to compare perceived Success between trainees and trainers

\begin{tabular}{|l|r|}
\hline & \multicolumn{1}{|c|}{ Success } \\
\hline Mann-Whitney U & 951.500 \\
\hline Wilcoxon W & 4692.500 \\
\hline Z & -.967 \\
\hline Asymp. Sig. (2-tailed) & .333 \\
\hline
\end{tabular}

\begin{tabular}{|l|l|r|r|r|}
\hline & Group & N & Mean Rank & \multicolumn{1}{c|}{ Sum of Ranks } \\
\hline \multirow{4}{*}{ Success } & Trainer & 25 & 60.94 & 1523.50 \\
\cline { 2 - 5 } & Trainees & 86 & 54.56 & 4692.50 \\
\cline { 2 - 5 } & Total & 111 & & \\
\hline
\end{tabular}

\section{CONCLUSION AND FUTURE RESEARCH}

It is concluded that mine rescue brigadesmen typically had positive learning experiences in 360-VR. Even though VR training is not common practice in the mining industry it appears to have been well received. Moreover, our findings confirm that even though fidelity is a factor usually considered by researchers to evaluate the effectiveness of VR-based training; however, it is controversial factor. There is no direct correlation between fidelity and learning and no clear indication of the level of fidelity required to achieve a successful VR-based training. Baker and colleagues (2005) observe that realistically rendered VR scenarios 
Pedram et al., The application of Simulation (Virtual Reality) for Safety Training in the Context of Mining Industry

don't contribute to a successful training as much as a realistic task and context which trainees can relate back to their workplace experience.

Also it has been identified that, there is a need for further research as:

- $\quad$ The current sample size was only 94 participants and only 85 has responded to all the questions. Thus, to be able to generalize our findings about VR as an industry training tool, larger sample size is needed.

- While the current study focused on the importance of the VR's technological features and the users' training experience, other factors were observed by the researcher to be important, such as i) the trainees' attitude toward the technology prior attending the training; ii) the fit of the technology for the particular training scenario; and iii) the industry's culture are important factors. To the best of our knowledge these three factors have received little empirical attention.

- Since 360-VR is being used as "safety training" tool it is almost impossible to be able to measure its success or transfer of training immediately as oppose to "operational training" where trainees performance can be measure straight after they are back to the mine. For safety training accidents must happen first and if it happened then brigades men performance might be measured. Therefore an alternative assessment strategy has been considered for future research.

\section{REFERENCES}

Burdea, G. \& Coiffet, P. (2003). Virtual reality technology. Presence: Teleoperators \& Virtual Environments, 12, 663664.

Deaton, J. E., Barba, C., Santarelli, T., Rosenzweig, L., Souders, V., Mccollum, C., Seip, J., Knerr, B. W. \& Singer, M. J. (2005). Virtual environment cultural training for operational readiness (VECTOR). Virtual Reality, 8, 156-167.

Fulton, E. A., Jones, T., Boschetti, F., Sporcic, M., Mare, W. D. L., Syme, G., Dzidic, P., Gortona, R., Little, L. \& Dambachera, G. (2001). A multi-model approach to stakeholder engagement in complex environmental problems. International Congress on Modelling and Simulation (MODSIM 2011), Perth, WA, 2011.

Jou, M. \& Wang, J. (2012). Investigation of effects of virtual reality environments on learning performance of technical skills. Computers in Human Behavior.

Kennedy, R. S., Lane, N. E., Berbaum, K. S. \& Lilienthal, M. G. (1993). Simulator sickness questionnaire: An enhanced method for quantifying simulator sickness. The international journal of aviation psychology, 3, 203-220.

Lucas, J. \& Thabet, W. (2008). Implementation and evaluation of a VR task-based training tool for conveyor belt safety training. ITcon.

Matthews, G., Joyner, L., Gilliland, K., Campbell, S., Falconer, S. \& Huggins, J. (1999). Validation of a comprehensive stress state questionnaire: Towards a state big three. Personality psychology in Europe, 7, 335-350.

Meadows, D. L. (2001). Tools for understanding the limits to growth: comparing a simulation and a game. Simulation \& Gaming, 32, 522-536.

Moreno, R. \& Mayer, R. (2007). Interactive multimodal learning environments. Educational Psychology Review, 19, 309-326.

Nutakor, D. (2008). Design and evaluation of a virtual reality training system for new underground rockbolters, ProQuest.

Patton, M. Q. (1997). Toward distinguishing empowerment evaluation and placing it in a larger context. Evaluation Practice, 18, 147-163.

Seymour, N. E., Gallagher, A. G., Roman, S. A., O’Brien, M. K., Bansal, V. K., Andersen, D. K. \& Satava, R. M. (2002). Virtual reality training improves operating room performance: results of a randomized, double-blinded study. Annals of surgery, 236, 458.

Taylor, G. S. \& Barnett, J. S. (2011). Training capabilities of wearable and desktop simulator interfaces. DTIC Document.

Tichon, J. \& Burgess-Limerick, R. 2011. A review of virtual reality as a medium for safety related training in mining. Journal of Health \& Safety Research \& Practice, 3, 33-40.

Van Wyk, E. \& De Villiers, R. (2009). Virtual reality training applications for the mining industry. Proceedings of the 6th international conference on computer graphics, virtual reality, visualisation and interaction in Africa, 2009. ACM, 53-63.

Witmer, B. G. \& Singer, M. J. (1998). Measuring presence in virtual environments: A presence questionnaire. Presence: Teleoperators and virtual environments, 7, 225-240. 